\title{
Report of the Round Table on Pathology and Experimental Transmission
}

\author{
(.hairman: R. J. W. Rees (UK); \\ Secretary: H. M. Portugal (Brazil); \\ Members: M. Bergel (Argentina); C. H. Binford (USA); \\ Y. 'T. Chang (US $\Lambda$ ); K. R. Chatterjec (India and UK); \\ J. Convit (Venczucla); W. H. Feldman (USA); W'. H. \\ Hadler (Brazil); N. Nishimura (Japan); J. M. Robson \\ (UK), Augusto Serial (Argentina); C. C. Shepard (USA); \\ F. l'. Wilkinson (Argentina).
}

'This report has been prepared by members of the round table group on pathology and experimental transmission and summarises the discussions held by this group in Rio de Janeiro on September 9- I . Our members fully endorse the value of the introduction of round table discussion groups and particularly in allowing time for these discussions before the main Ciongress.

\section{PATHOLOGY}

Because pathology was included as a special subject for the first time at the 8th Intcrnational Congress of I.cprology, 'Tokyo, it was revicwed then very broadly by the 'Technical Committee on Bacteriology and Pathology. 'Therefore, our present report deals more specifically with aspects of pathology in which progress has bcen made in the last five years or where we consider views expressed by the previous Technical Committec should be reiterated.

\section{Practical application of pathology}

Histological examination of biopsy material is an essential examination for the proper diagnosis, classification and prognosis of the disease and for assessing the progress or regression of the disease in patients under treatment. Between the two polar groups of leprosy there is a wide spectrum of innumerable intermediate responses which are dependent on the host-parasite reaction. Histological examination provides an essential picture of this host-parasite relationship and should be given at least equal weighting with the clinical picture. In properly conducted chemotherapcutic trials it is important to select suitable cases based on both histological and clinical examination in order to exclude where possible patients with a more favourable prognosis. Because histology provides an overall picture of the cellular and bacillary elements the examination can be used to assess both the lesion size (area of cellular infiltration) and the concentration of acid-fast bacilli. Combination of these two assessments into a so-called 'histological' index provides a more accurate 
assessment of the infection than the classical bacteriological index. 'Thus a series of biopsies taken from the same site or sites during the course of treatment can provide an additional important measure of progress. Pathologists are urged again to seek agreement on the application of this histological index for the careful assessment of progress of patients in chemotherapeutic trials.

ow that there is a considerable weight of evidence to support the view that irregularly stained bacilli are degenerate, the reporting of the morphological appearance (irregularly stained and solidly stained organisms) of bacilli in Ziehl-Neclsen stained sections has added importance. It has obvious applications in recording the progress to treatment in chemotherapeutic trials. More detailed knowledge of the proportions of irregularly and solidly staining bacilli in different types of leprosy, during reactions and in different sites in the body or in particular types of cells, would help in elucidating the pathogenesis of leprosy.

Attention is drawn to the value of using a simple histochemical test (Sudan III Stain) on frozen sections from diagnostic biopsies. Lipids are found in lepromatous and dimorphous lesions but not in tuberculoid.

The Committee appreciated the recent contribution of DR. WADE in sending to members of the pancl and others a set of excellently prepared and stained slides illustrating the histopathology of I2 lesions of the 'Histioid variety of lepromatus leprosy'. There was insufficient time to adequately study the slides but the Committee urge those who received the slides to send their comments directly to DR. WADE.

\section{Recent advances in pathology}

\section{A. The identification of "fuchsino}

given by Khanolkar to round and spindle-shaped cells containing acidfast granules in sections stained by the Zichl-Neclsen method. More recently it has been shown that these fuchsinophil cells are tissue mast cells commonly found in most tissues, including the dermis, of normal healthy subjects. Although the granules are acid-fast they show also the characteristic metachromasia of mast cells.

B. Neuropathology. Leprosy holds a special challenge to neuropathology because it is the only mycobacterial infection in man which involves nerves. Because, in fact, leprosy has a predilection for peripheral nerves it is more than likely that research in the field of ncuropathology will throw new light on the pathogenesis of leprosy. Advances during the last five ycars based on carcful observations (using light and clectron microscopy) of human leprosy tissues and on experiments in animals indicate that Myco. leprae are found usually in the Schwann cells. These observations contradict the view of the pathogenesis of leprosy put forward by Khanolkar in which the nerve fibre is considered to be the site in which the bacilli multiply and in which the bacilli travel along the nerve pathway in the axoplasm of the nerve fibre.

Recent experimental studies in rats and in man have shown that Schwann cells behave as phagocytes capable of ingesting not only neural 
debris but also forcign particles, such as carbon particles or mycobacteria, in their neighbourhood. It is significant that Schwann cells have becn shown to have general phagocytic activity as well as the well recognised function of phagocytosing neural debris. 'The Schwann cells are most phagocytic at the time of nerve degeneration or regeneration. Since there is a physiological turnover of nerves all the time, particularly in the skin, there will always be some Schwann cells in an active phase. Some recent experiments(*) on patients with leprosy have shown that these activated Schwann cells have selective powers of ingesting mycobacteria since they actively phagocytosed heat-killed human leprosy but not heat-killed rat leprosy bacilli. 'This observation is of such fundamental importance that it must be confirmed. In addition to these basic observations on the nerve fibre-Schwann cell complex in man and experimental animals, it is of interest that nerve involvement has been observed also in some of the experimental mycobacterial infections in animals following the injection of Myco. leprae.

C. Application of Electron Microscopy. In addition to the value of electron microscopy for defining more precisely nerve involvement in leprosy it is a valuable tool for examining the host-bacillus relationship at the cellular level. Recent studies suggest that the intra-cellular reaction to Myco. leprae differs in lepromatous and borderline patients and furthermore degenerate bacilli predominate in Virchow cells.

\section{EXPERIMENTAL TRANSMISSION}

'The members of this Round table committec are favourably impressed by the recent progress which has been made in the field of experimental transmission of Myco. leprae. At present some of the results obtained in different laboratories using different conditions or species of animals are not the same. It is considered that the more progressive and extensive experimental infections in animals need to be investigated further and particularly to be confirmed more consistently. and in different laboratories, before finally accepting them as being caused by the human leprosy bacillus. Nevertheless the committec consider that the definite infection produced in the foot-pads of mice is presumably a human leprosy infection, particularly since it has now been reproduced consistently in several different laboratorics and with Myco. leprae derived from patients all over the world.

In order to maintain progress in this most important field, to encourage exchange of data and materials and to stimulate others to undertake this type of work, this committee recommends that the ILA set up a working committee on Experimental 'Transmission.

\section{A P P E NDIX}

The members of this group considered that it was of the greatest importance at the present stage of transmission studies to summarize their views 
regarding the problems and methods in animal transmission experiments and the criteria for assessing successful claims.

Inoculum: Suspensions of Myco. leprac from man

(a) Biopsy from untreated smear positive patients (to include all types of leprosy from lepromatous to reactional tuberculoid - classified, if possible, clinically and histologically.) Biopsies should not be taken from ulcerated lesions.

(b) Suspension of bacilli from biopsy to be prepared and inoculated into animals as soon as possible (if delay is unavoidable tissues to be kept in wet and not dry ice). Suspension to be prepared in saline or albumin/ saline. Compare suspensions of bacilli prepared with and without separation of human tissuc by differential centrifugation.

(c) Count number of acid-fast bacilli in suspension (Hanks'; Hilson and Elek, method) in order to inoculate animals with known numbers of bacilli and compare large and small inocula. Carry out a differential count of the proportion of uniformly and irregularly staining bacilli.

(d) Culture suspension on types of media suitable for isolating mycobacteria and incubate for not less than three months at two temperatures, $37^{\circ} \mathrm{C}$ and $30^{\circ} \mathrm{C}$ to $34^{\circ} \mathrm{C}$. Suspensions to be cultured untreated and also after decontamination by a method used for treating sputum. (If culture positive mycobacteria are isolated they should be identified and inoculated into animals.)

\section{Cihoice of animals and plan of animal experiments}

(a) Present results suggest the use of the mouse and hamster, but as many different species of animals as possible should be compared. 'The use of very young animals was recommended. Because of the slow develop)ment of human leprosy animals with a long life span should be tried. It was particularly recommended that an attempt be made to inoculate a colony of young chimpanzecs and follow these by annual biopsy throughout their life span. Where possible a study should be undertaken comparing results in differcnt strains of in-bred and pure-line mice; although every routc and site of inoculation should be studied, particular attention should be paid to colder sites, i.e., ear, foot-pad and testis. (In order to compare results in different centres the animal house temperature and the type of dict should be recorded.)

In addition to the usc of normal animals it was recommended that the effect of hormones, diet (particularly including pro-oxidant diets) $\mathrm{X}$ irradiation and any other alterations which might increase susceptibility to infection should be studicd.

(b) Controls :

I. Inoculation of living bacilli

2. Inoculation of heat-killed bacilli

*3. Group of uninoculated normal animals

*4. Group of uninoculated modified animals, i.e., special diet, etc. 
5. Where specially modified groups of animals are used they should be divided into two groups receiving living and heat-killed bacilli

6. Small group of standard diagnostic animals, i.e., rabbit and guinea pig.

*'l'hese groups are of the greatest importance because of the observation that normal stocks of mice and hamsters have been shown to harbour non-culturable ('Murine leprosy type') mycobacteria.

\section{Investigation of bacteria isolated from the animals}

(a) Count the total number of acid-fast bacilli harvested from the animal or particular site of inoculation and determine the proportion of uniformly and irregularly stained bacilli.

(b) Culture bacilli isolated (as per section - Inoculum (d) above).

(c) Preparation of a standard type lepromin (Integral and/or Dharmendra type) from the bacilli harvested from the animals and to be compared with a similarly prepared human lepromin in patients with tuberculoid and lepromatous leprosy. The tests to be carried out and read 'blind'. ('The 'animal' and human lepromins to be adjusted to contain the same number of acid-fast bacilli. Since the 'animal' lepromin will contain animal tissue antigen it is possible that this foreign antigen could produce a skin reaction in man and it may be necessary to skin test with a 'lepromin' prepared from normal animal tissue or add normal animal tissue to the standard human lepromin.)

(d) Enzymatic Studies.

(e) Serological Studies.

\section{Histological examination of the animal tissues}

This study should be undertaken by a histopathologist trained in leprosy who should not only look for alterations similar to those in man but also for any unusual findings, particularly those recurring in animals. Histological examination should be carried out as a routine on all animal tissues (Zenker type fixative recommended $-\mathrm{H}$ and $\mathrm{E}$ and $\mathrm{ZN}$ (Wade modifications). If tissues have to be sent away use formalin fixative).

Because acid-fast bacilli have been found associated with nerves in a number of the recent experimental leprosy infections these should be observed and where possible a special study be undertaken using light (including silver staining) and electron microscopy. Because in man leprosy is the only mycobacterial infection known to involve nerves and although it is stated that murine leprosy does not affect nerves, the Committee recommended that this question must be re-investigated in murine leprosy.

\section{Response of the experimental infection to drugs}

The pattern of response of the infection in animals to anti-mycobacterial drugs should be studied as a means of: (I) identifying the organism, and (2) screening new anti-leprosy drugs. 
All the above studies are undertaken in order to determine how far the experimental leprosy infection resembles the infection in man and to exclude where possible any other known mycobacterial infection, particularly the non-cultivatable murine leprosy infection.

It is important to include adequate controls and positive results should only be accepted if they are consistent and reproducable.

\section{Round Tableon Pathology and Experimental Trans- mission: Memorandum on the Examination of the Morphology of M. Leprae in the Study of Leprosy}

One of the most characteristic features of a smear of human leprosy bacilli stained by the Zichl-Neelsen method is the presence of a significant proportion of organisms which stain irregularly with carbol-fuchsin. Hansen was the first to suggest that irregular staining indicated a degenerative change in the bacilli. The use of sulphones, where clinical improvement is associated with a significant increase in the proportion of irregularly stained bacilli ( $5^{\text {th }}$ Int. Congress Lcprology, Havana, I948), has strengthened Hansen's original suggestion.

More recently detailed studies, using electron microscopy, on the morphology of live and dead murine leprosy bacilli and other bacteria (including $E$. coli) have shown conclusively that death of these organisms leads to the loss of cytoplasmic content and that in the case of mycobacteria (including $M$. lepraemurium and $M$. leprae) these important morphological changes are manifest as irregular staining of the bacilli using the Zichl-Neelsen method. It has been established in studies on $M$. lepraemurium that only the solid staining bacilli, the so-called 'solid staining' forms, are viable and all forms of irregularly stained bacilli whether defined as 'fragmented' or 'granular' are dead organisms. On this analogy, and on the evidence of electron microscopy, it is reasonable to assume that the same can be said of human leprosy bacilli, and this Committee wishes to draw the attention of the 8th International Congress of Leprology to the importance of studying the morphology of the bacilli as a routine procedure in assessing the progress of leprosy patients. In order to obtain uniform results the following suggestions are made:

I. Any of the standard Ziehl-Neelsen staining methods can be used and satisfactory definition is obtained by using an oil immersion lens with $\mathrm{x} 6$ eye-piece for examining the smears.

2. It is important to prepare thin smears and to exclude red cells.

3. Morphology should be reported only on well defined single bacilli. Morphology should not be attempted on organisms in globi or in clumps.

4. Morphology, where possible, should be determined on oo or more 
organisms in fields picked at random over the smear, and reported as follows:

(a) Results given as the proportion of uniformly or 'solid' stained bacilli, i.e., oo 'solid' staining bacilli in an examination of $\mathrm{s} 00$ organisms $=10 / 100$, ctc.

(b) Only organisms showing uniform staining with carbol-fuchsin down their length should be classified as uniformly or solid staining organisms. Bacilli containing dense bodies, often polar, and giving the appearance of a club are frequently seen in an otherwise uniformly stained organism. Such bacilli are classified with the uniformly stained forms.

(c) Every degrec of irregular staining ('gramular' or 'fragmented', etc.) with carbol-fuchsin should be classified as irregularly stained organisms. lery short uniformly stained bacilli are classified with the irregularly stained forms.

'The examination of the morphology of human leprosy bacilli has several important applications:

I. For following the response to chemotherapy, particularly in the early phase and also for detecting detcrioration, possibly indicating the emergence of drug resistance (indicated as an increase in the proportion of uniformly staining bacilli).

2. For studying the effect of 'reactions' on the progress of leprosy.

3. A general application of the method for all bacteriological studies on leprosy, particularly in those concerned in studies on the different types and stages of the discase and for determining the persistance of healthy bacilli in different tissues. Although the original observations were made on stained smears, the same technique can be applied also to stained sections of tissuc. 